\title{
Multi-Layer Perceptron with Positive and Negative Pulse Glial Chain for Solving Two-Spirals Problem
}

\author{
Chihiro Ikuta \\ Yoko Uwate \\ Yoshifumi Nishio \\ Dept. of Electrical and Electronics Eng., Dept. of Electrical and Electronics Eng., Dept. of Electrical and Electronics Eng., \\ Tokushima University \\ 2-1 Minami-Josanjima, \\ Tokushima Japan \\ Tokushima University \\ 2-1 Minami-Josanjima, \\ Tokushima Japan \\ Tokushima University \\ 2-1 Minami-Josanjima, \\ Tokushima Japan \\ Email: ikuta@ee.tokushima-u.ac.jp \\ Email: uwate@ee.tokushima-u.ac.jp \\ Email: nishio@ee.tokushima-u.ac.jp
}

\begin{abstract}
A glia is a nervous cell existing in a brain. The brain is composed of the relationship with glias and neurons. By an ion concentration, the glia transmits signal to neurons and neighboring glias. In this study, we propose the MLP with positive and negative pulse glial chain which is inspired from features of the biological glia. We add the MLP to the positive and negative pulse glial chain. In the positive and negative pulse glial chain, the glias are connected to the neurons one by one. The glia generates pulse when the glia is excited by the connected neuron's output. If the connected neuron has large amount of output, the glia generates positive pulse. Moreover, if the connected neuron has small amount of output, the glia generates the negative pulse. The positive and negative pulse are propagated to the connected neuron and neighboring glias. We consider that the positive and negative pulse glial chain give the relationships of position of neurons in a same layer. By solving a Two-Spirals Problem (TSP), we confirm that the proposed MLP has better a learning performance and a generalization capability than the conventional MLP.
\end{abstract}

\section{INTRODUCTION}

Nervous cells compose a higher brain function. The nervous cells are divided into two kinds of cells which are neurons and glias. Many researchers have researched about biological features and its applications of the neurons. The neurons transmit electric signals to each other, its work composes the thinking, memories, and others. The glia had not been noted, because the activity of the glia in the brain could be not easily described. Thereby, this cell is known to statistic and support cell for a long time. However, some researchers discovered that the glias transmit signals by change of concentrations of several ions [1]. The glia has many receptors of ions which are an adenosine triphosphate (ATP), a glutamate acid (Glu), a calcium ion $\left(\mathrm{Ca}^{2+}\right)$, and so on, moreover, this cell generates $\mathrm{Ca}^{2+}$ concentration wave [2][3]. These ions are important for the brain works, actually, the neuron uses the ATP and the Glu in the gap junction [4]-[6]. We have noticed $\mathrm{Ca}^{2+}$, because $\mathrm{Ca}^{2+}$ concentration wave influences the membrane potential of the neurons [7][8]. Moreover, we consider that the features of glia can be applied to artificial neural networks.

Various kinds of artificial neural networks and its applications have been proposed. Multi-Layer Perceptron (MLP) which is proposed by D.E. Rumelhart is one of the artificial neural networks [9]. The MLP is composed of the layer of neurons. We can obtain the expectation relationships between inputs and outputs by tuning weights of connections. Back Propagation algorithm (BP) is often used to the tuning algorithm for the weight of connections. The MLP has the connections and the relationships between neurons in different layers. However, the neurons do not connect in a same layer. We noticed this fact and consider that we can give the relationships in the same layer by the network of glias.

In this study, we propose a MLP with positive and negative pulse glial chain. The positive and negative pulse glial chain is inspired from the features of the biological glia. All glias one-by-one connect to the neurons in hidden layer and influence each other. When the neuron generates large output, the connecting glia is excited and generates positive pulse. When the neuron generates small output, the connecting glia is excited and generates negative pulse. The excitation glia generates pulse which influence neuron threshold and the excitation of neighboring glias. The output of glia is attenuated in an exponential fashion. Moreover, the glia has a period of inactivity. If the neighboring glia or the connected neuron affect the excitation glia, the glia cannot be excited again during the period of inactivity. We consider that the propagation of glias' pulses give the relationships of neurons in the same layer. We believe that the relationships of neurons in the same layer improve the MLP learning performance. By the computer simulation, we confirm that the MLP with positive and negative pulse glial chain has better learning performance than the conventional MLP.

\section{Multi-Layer Perceptron With positive and negative pulse GLIAL CHAIN}

The MLP is the most famous feed forward neural network. It is composed of the layers of neurons. We can change the output of the network by tuning the weights of connections between neurons. We often use the BP algorithm to decide of weights of connections. In the MLP, the neurons connect with other neurons in other layer. The MLP can be applied to a pattern classification, a pattern recognition, a data mining, and so on. However, it does not have relationships between neurons in the same layer. When we consider a biological system, the neuron works have existing the position relationship. We 
notice a glia and assume that the glia gives the position relationship to the neurons. We connect the glia to the neurons in the hidden layer which is shown in Fig. 1.

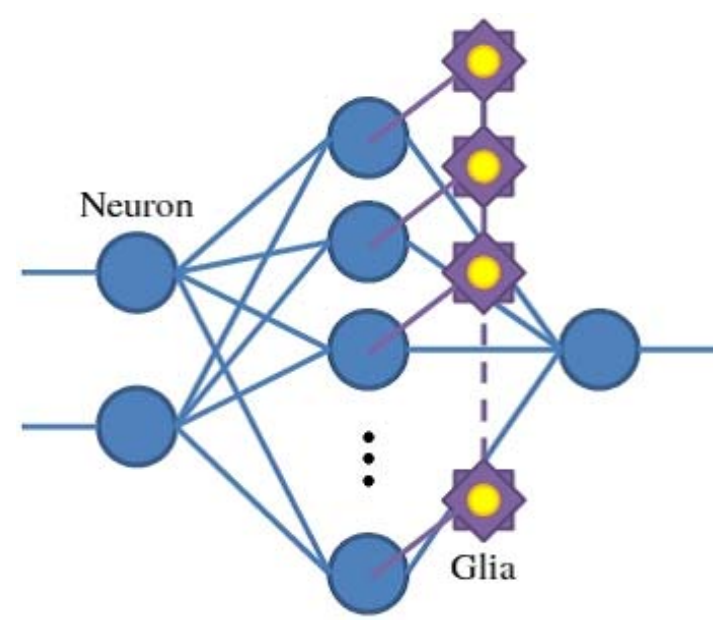

Fig. 1. MLP with positive and negative pulse glial chain.

\section{A. Glial pulse chain}

The glia is one of nervous cells existing in a brain. For a long time, the glia had not been investigated in detail, because this cell was considered that it could not transmit signals similar to neurons. However, some researchers discovered that the glia transmits signal by several ions concentrations. The glia has many ion receptors, for example, ATP, Glu, GABA, $\mathrm{Ca}^{2+}$, and so on. These ions are used by the signal of neuron in the gap junction, thereby the glia is known that has relationships with neuron signals. We notice the $\mathrm{Ca}^{2+}$ concentration, because the glia generate $\mathrm{Ca}^{2+}$ concentration wave and it is propagated to wide range in the brain. Moreover, the $\mathrm{Ca}^{2+}$ affects a membrane potential of the neuron.

In this paper, we propose the positive and negative pulse glial chain which is inspired from the biological features of glia. The glias are connected to neurons one-by-one. When the connected neuron has a large output, the glia is excited and generates the positive pulse. When the connected neuron has a small output, the glia generates the negative pulse. The excitation glia generates the pulse. This pulse affects neighboring glias and the threshold of connected neuron. We show an example of propagation of glial effect in Fig. 2. One neuron generates large output and excites the connecting glia. Next, the excitation glia generates pulse. After that, the pulse affects neighboring glias and the connected neuron. The glia which the pulse received is excited, and generate pulses. Then if the first excitation glia receive the large amount output of neuron, the glia cannot excite again. Because the glia has a period of inactivity. The glias repeat these works and propagate the glial effects.

The glia has two different states which are the positive response and the negative response. We define the output

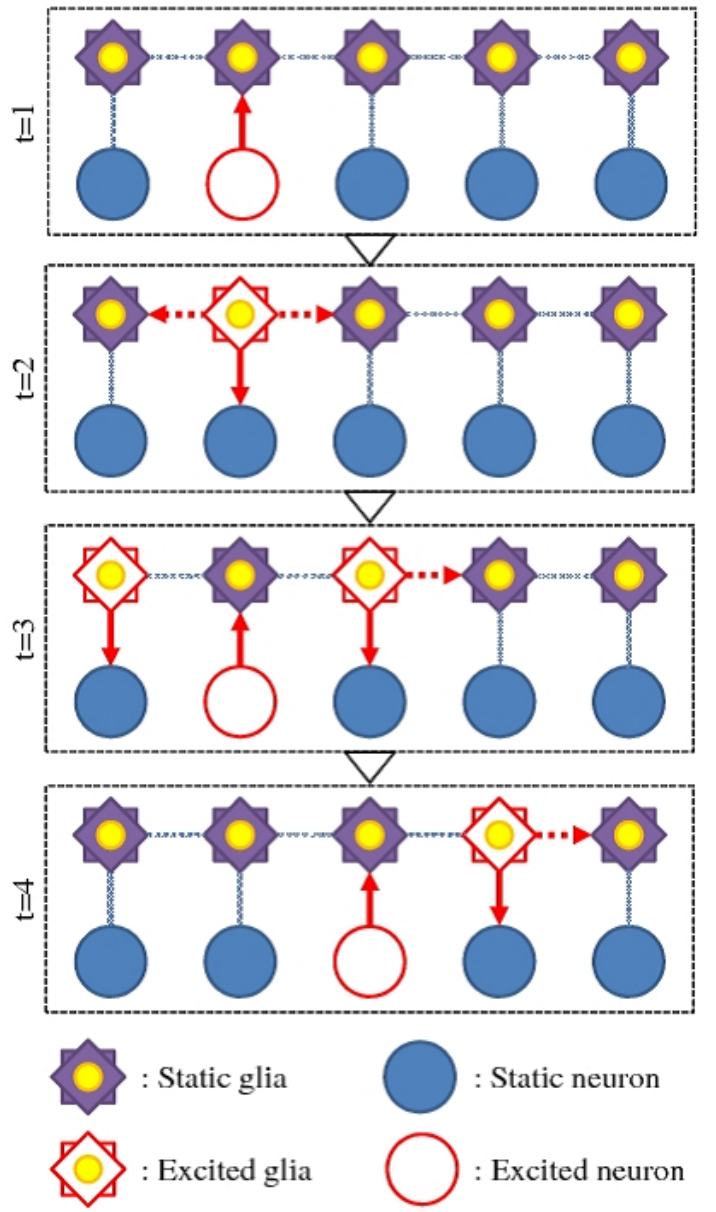

Fig. 2. Pulse propagation.

function as the positive response of the glia in Eq. (1).

$$
\begin{aligned}
& \psi_{i}(t+1)= \\
& \left\{\begin{array}{l}
1, \quad\left\{\left(\theta_{n}<y_{i} \cup \psi_{i+1, i-1}(t-i * D)=1\right)\right. \\
\gamma \psi_{i}(t), \text { else, }
\end{array}\right.
\end{aligned}
$$

where $\psi$ is an output of a glia, $\gamma$ is an attenuated parameter, $y$ is an output of a connected neuron, $\theta_{n}$ is a glia threshold of excitation, $\theta_{g}$ is a period of inactivity, and $D$ is a delay time of a glial effect. Moreover, we define the output function as the negative response of the glia in Eq. (2).

$$
\begin{aligned}
& \psi_{i}(t+1)= \\
& \left\{\begin{array}{lr}
-1, & \left\{\left(1-\theta_{n}>y_{i} \cup \psi_{i+1, i-1}(t-i * D)=1\right)\right. \\
\gamma \psi_{i}(t), & \text { else, }
\end{array}\right.
\end{aligned}
$$

The glia does not learn, it depend on the output of connected neurons. However, the neurons are learned by BP algorithm, thus the generation pattern of glia output can dynamically change during the learning. Figure 3 is an example of generation pulses as 10 glias. At first, the 4th glia, the 6th glia, and the 9th glia are excited by the connected neurons. There pulses 
propagate to neighboring glias. According to the passage of time, the generation pulse pattern is changed. Actually, the generation pulse pattern change from (a) to (b). Because the outputs of neurons are changed by the learning. We can say that the glial effect change the neurons threshold, thereby the glias influence the leaning of the MLP.

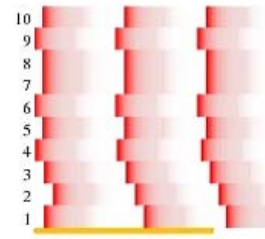

(a)

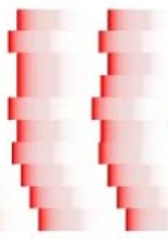

个

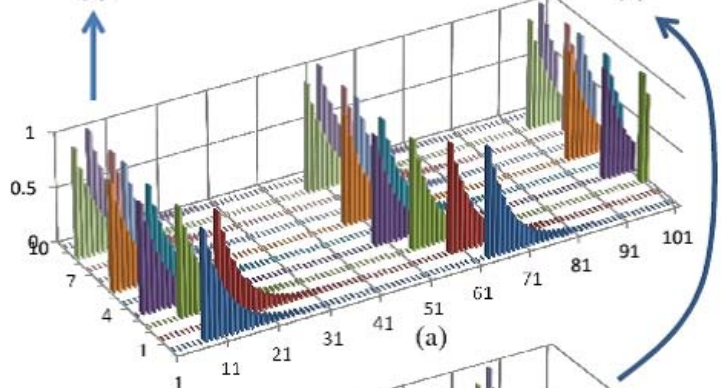

1

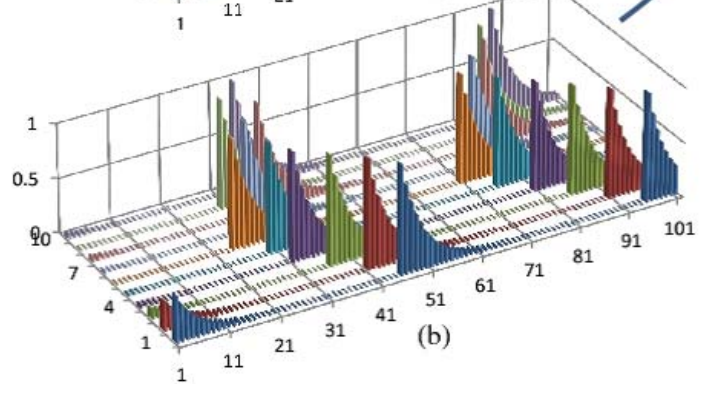

Fig. 3. An example of glial pulses $(D=5)$.

\section{B. Updating rule of neuron}

The neuron has multi-inputs and single output. We can change the neuron output by the tuning the weights of connections. The standard updating rule of the neuron is defined by Eq. (3).

$$
y_{i}(t+1)=f\left(\sum_{j=1}^{n} w_{i j}(t) x_{j}(t)-\theta_{i}(t)\right),
$$

where $y$ is an output of the neuron, $w$ is a weight of connection, $x$ is an input of the neuron, and $\theta$ is a threshold of neuron. Next, I show a proposed updating rule of the neuron. We add the glial effect to the threshold of neuron. This updating rule is used to neurons in the hidden layer. It is described by Eq. (4).

$$
y_{i}(t+1)=f\left(\sum_{j=1}^{n} w_{i j}(t) x_{j}(t)-\theta_{i}(t)+\alpha \psi_{i}(t)\right),
$$

where $\alpha$ is a weight of the glial effect. We can change the glial effect by change of $\alpha$. In this equation, the weight of connection and the threshold are changed by BP algorithm as same as the standard updating rule of the neuron. However, the glial effect is not changed. It is updated by Eq. (1).

Equations. (3) and (4) are used a sigmoidal function to an activating function which is described by Eq. (5).

$$
f(a)=\frac{1}{1+e^{-a}}
$$

where $a$ is an inner state.

\section{Simulations}

In this section, we show the experimental result. We use seven kinds of the MLPs for comparison of the performance.

(1) The conventional MLP

(2) The MLP with random noise

(3) The MLP with random timing pulses

(4) The MLP with same timing pulses

(5) The MLP with pulse glial chain (only propagation to one directions)

(6) The MLP with pulse glial chain

(7) The MLP with positive and negative pulse glial chain.

The MLP with random noise is given an uniformed random noise to the threshold of the neurons in the hidden layer. The MLP with random timing pulses receive the pulses at random timing to the the threshold of the neurons. In the MLP with random timing pulses, all glias are independent from other glias. The MLP with same timing pulses receive the pulse at same timing. The all glias are excited and generate pulses at same time. These pulses are given to the threshold of connected neurons at same time. In the MLP with pulse glial chain (only propagation to one directions), the glias can only propagate the pulses to one side. In the MLP with pulse glial chain, the glias have only positive response.

We use a Mean Square Error (MSE) to the error function. It is described by Eq. (6).

$$
M S E=\frac{1}{N} \sum_{n=1}^{N}\left(T_{n}-O_{n}\right)^{2},
$$

where $N$ is a number of learning data, $T$ is a target value, and $O$ is an output of MLP. We obtain results which are an average error, a minimum error, a maximum error, and a standard deviation of the results.

\section{A. Simulation task}

In this simulation, we use the Two Spirals Problem (TSP). The TSP is a famous benchmark of the artificial neural network [10][11]. It is known to a high nonlinearly task. This task has the two different spiral points. The MLPs learn the classification of two spiral points. We use the two different tasks which spirals are composed of 98 points and 130 points. Figure 4 is two spirals for different number of points. The MLPs learn each coordinates of points. Moreover, we can obtain the generalization capability from solving the TSP. We input coordinates between 0 and 1 to the after learning MLP. We obtain the output of the network and can know that each coordinate fits into which spirals. Figure 5 shows that 
the ideal results of the classification of coordinates. We make the ideal classification result by calculation of norm between coordinates and spiral points. In this simulation, the MLP is composed of 2-40-1. We increase the number of neurons in the hidden layer. Because, the learning performance of the MLP is improved by the number of neurons.

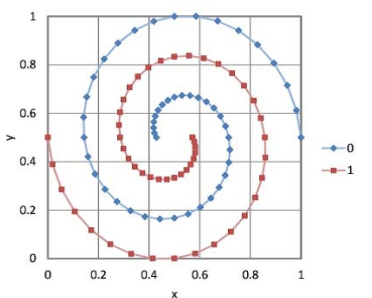

(a) 98 .

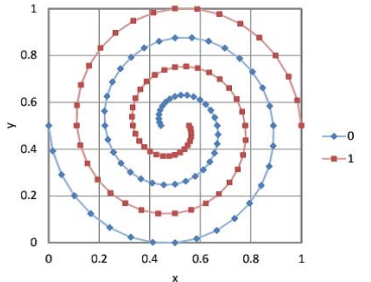

(b) 130
Fig. 4. Target points.

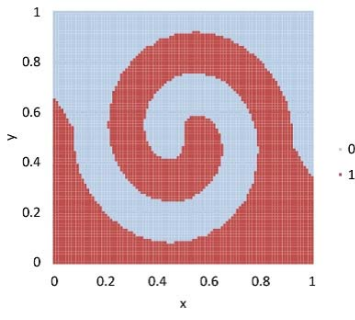

(a) 98 .

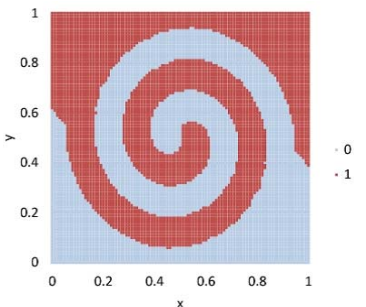

(b) 130 .
Fig. 5. Ideal classification results.

1) Spirals composed of 98 points: First, we show the experimental result from the learning 98 points in Table I. The minimum error is similar value. From this result, every MLP can search the optimum solution. Thus, the average error shows the escaping performance from local minimum. The average error of the MLP with negative and positive pulse glial network is the best of all. When we compare the conventional MLP and proposed MLP, the proposed MLP has twice the learning performance.

TABLE I

LEARNING PERFORMANCE.

\begin{tabular}{ccccc}
\hline & Average & Minimum & Maximum & Std. Dev. \\
\hline$(1)$ & 0.04153 & 0.00017 & 0.18387 & 0.02637 \\
$(2)$ & 0.03711 & 0.00006 & 0.17352 & 0.02946 \\
$(3)$ & 0.03666 & 0.00015 & 0.08208 & 0.02195 \\
$(4)$ & 0.03873 & 0.00022 & 0.17335 & 0.02632 \\
$(5)$ & 0.03178 & 0.00024 & 0.07186 & 0.01986 \\
$(6)$ & 0.02072 & 0.00011 & 0.08192 & 0.01782 \\
$(7)$ & 0.01531 & 0.00009 & 0.06157 & 0.01636 \\
\hline
\end{tabular}

Next, we show that the statistic classification result in Table II. In this result, the average of error is the smallest when we use the MLP with positive and negative pulse glial chain. Generally, the generalization capability become weak when the MLP has over learning. The MLP with positive and negative pulse glial chain has both the performance of function approximation and the performance of the generalization capability. From this result, the proposed MLP has an ability of searching global solution.

TABLE II

Classification Performance.

\begin{tabular}{ccccc}
\hline & Average & Minimum & Maximum & Std. Dev. \\
\hline$(1)$ & 0.15029 & 0.08085 & 0.21127 & 0.02434 \\
$(2)$ & 0.13966 & 0.08083 & 0.20378 & 0.02879 \\
$(3)$ & 0.14702 & 0.07965 & 0.20083 & 0.02553 \\
$(4)$ & 0.15081 & 0.07601 & 0.22355 & 0.02745 \\
$(5)$ & 0.13123 & 0.07986 & 0.17376 & 0.02162 \\
$(6)$ & 0.12233 & 0.08140 & 0.17042 & 0.01939 \\
$(7)$ & 0.10980 & 0.06408 & 0.15069 & 0.01902 \\
\hline
\end{tabular}

Figure 6 is examples of the classification results. We show the near results of the average. The results of the MLP with pulse glial chain and the MLP with positive and negative pulse glial chain can represent the two spirals. However, the others become similar, and are cut a part of spirals. We can see that the boundary line of the spirals as the MLP with positive and negative pulse glial chain is smoother than the MLP with pulse glial chain. We consider that the MLP with positive and negative pulse glial chain has better classification ability than the MLP with pulse glial chain.

2) 130 spirals: In this section, we show that the result for the MLP learning spirals of 130 points. Table III is the experimental result of the MLPs. The results similar trend to the 98 points. The error average of the conventional MLP becomes large value. In the TSP, when the spirals points increase, the task become difficult. Thus, the conventional MLP is trapped local minimum. The noise is efficiency for the local minimum problem. The noise gives the energy to the MLP, and we believe that the MLP escapes from the local minimum. From this table, we can see that the noise pattern is important for the MLP learning. The noise patterns of (2), (3) and (4) is little efficiency for the learning performance in this simulation. All MLPs with pulse glial chain decrease the error. From this result, we consider that the relationship of position of neurons is important for the MLP learning. This because of that the MLP with pulse glial chain is better the learning performance than the MLP with pulse glial chain (only one direction). Moreover, in the MLP with positive and negative pulse glial chain, the glial effects are decomposed which are the positive part and the negative pulse part. We consider that the positive pulse part and the negative pulse part accentuate the relationship of positions of neurons.

TABLE III

LEARNING PERFORMANCE.

\begin{tabular}{ccccc}
\hline & Average & Minimum & Maximum & Std. Dev. \\
\hline$(1)$ & 0.12269 & 0.00831 & 0.23857 & 0.05554 \\
$(2)$ & 0.10847 & 0.00047 & 0.24278 & 0.05742 \\
$(3)$ & 0.10858 & 0.00416 & 0.26844 & 0.05546 \\
$(4)$ & 0.09391 & 0.00602 & 0.24616 & 0.05276 \\
$(5)$ & 0.04597 & 0.00052 & 0.12401 & 0.02537 \\
$(6)$ & 0.03830 & 0.00063 & 0.12190 & 0.02589 \\
$(7)$ & 0.01673 & 0.00048 & 0.08527 & 0.01661 \\
\hline
\end{tabular}

Table IV is the statistic classification result. The MLP with 


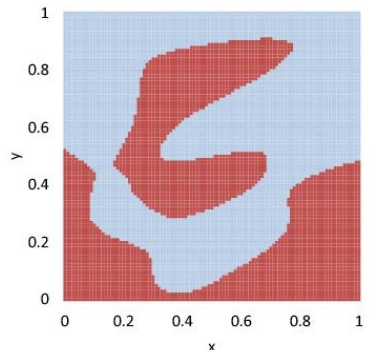

(a) Conventional. MLP

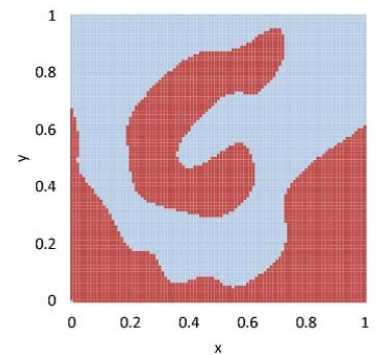

(c) MLP with random timing.

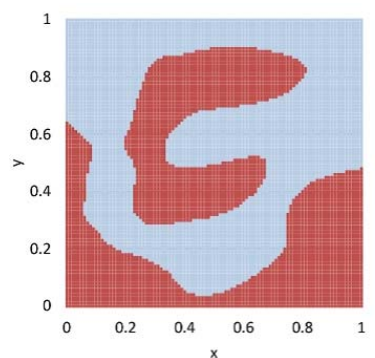

(e) Proposed MLP (one direction).

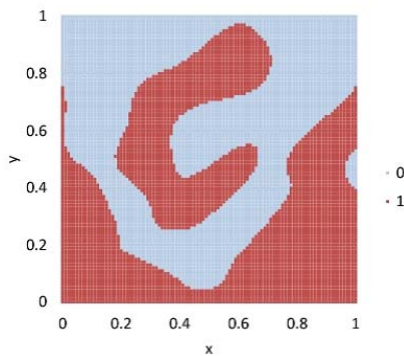

(b) MLP with random noise

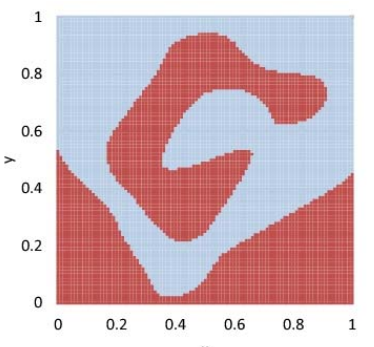

(d) MLP with same timing.

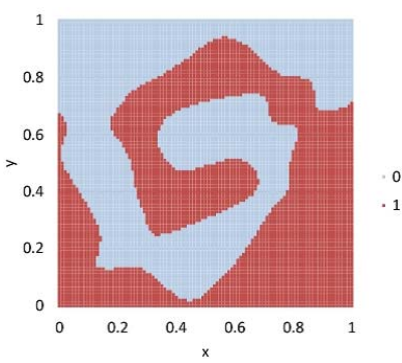

(f) Proposed MLP.

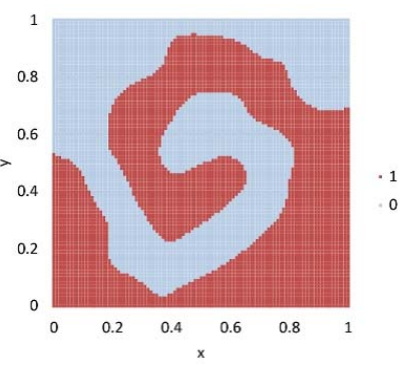

(g) Proposed MLP.

Fig. 6. Examples of classification results.

positive and negative pulse glial chain is the best of all. This error average is similar to the learning 98 points.

Finally, we show the examples of classification results near the error average in Fig. 7. The conventional MLP cannot represent the two spirals. Figures 7 (b), (c) and (d) look like to represent the spirals. However, they are cut some parts of spirals. Figure 7 (e) is cut two parts of spirals. Figure 7 (f) is cut one part of spirals. We can see the two spirals in these two classification results. The MLP with positive and negative pulse glial chain can almost represent the two spiral. This image is not cut any part. From this simulation, we consider that the MLP with positive and negative pulse glial chain has the highest generalization capability of all MLPs.

TABLE IV

Classification performance.

\begin{tabular}{ccccc}
\hline & Average & Minimum & Maximum & Std. Dev. \\
\hline$(1)$ & 0.21782 & 0.10565 & 0.29477 & 0.03858 \\
$(2)$ & 0.19278 & 0.10460 & 0.33065 & 0.04434 \\
$(3)$ & 0.19799 & 0.12332 & 0.34693 & 0.04086 \\
$(4)$ & 0.19564 & 0.11603 & 0.30857 & 0.03657 \\
$(5)$ & 0.16068 & 0.08651 & 0.22352 & 0.03080 \\
$(6)$ & 0.14731 & 0.08792 & 0.23723 & 0.02826 \\
$(7)$ & 0.11925 & 0.06585 & 0.18821 & 0.02324 \\
\hline
\end{tabular}

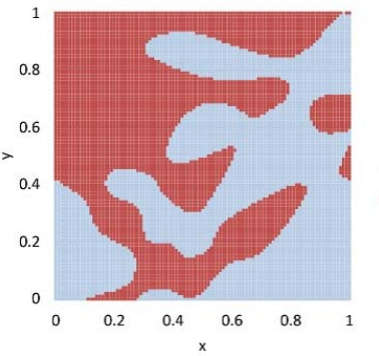

(a) Conventional. MLP

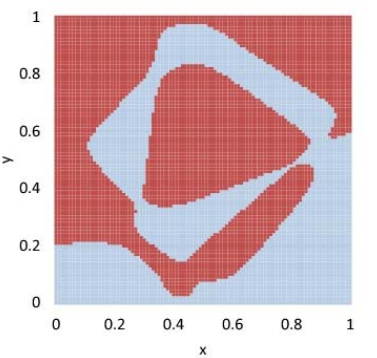

(c) MLP with random timing.

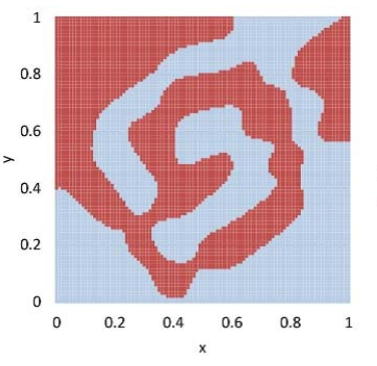

(e) Proposed MLP (one direction)

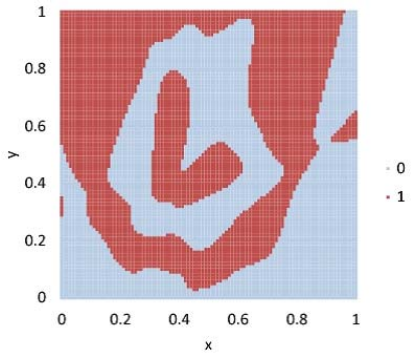

(b) MLP with random noise

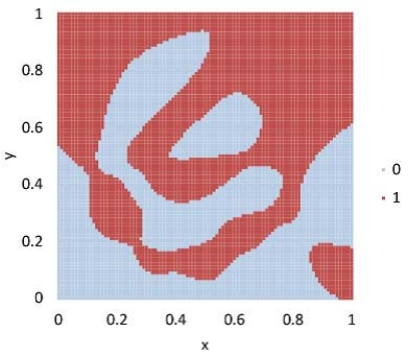

(d) MLP with same timing.

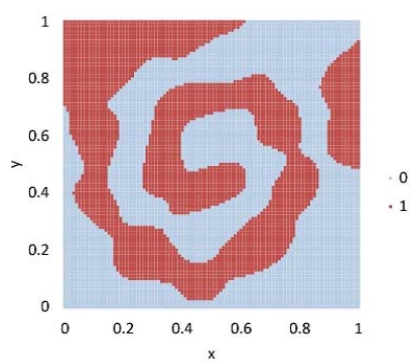

(f) Proposed MLP.

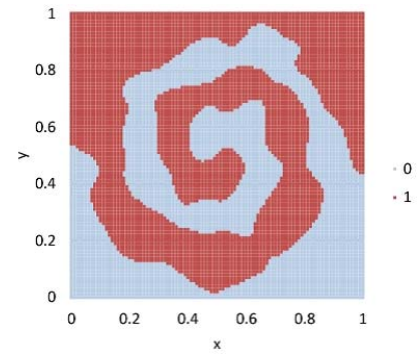

(g) Proposed MLP.

Fig. 7. Examples of classification results. 


\section{Conclusion}

In this study, we have proposed the MLP with positive and negative pulse glial chain which is inspired from the feature of biological glia. We add the pulse glial chain to the neurons of the hidden layer in the MLP. In the positive and the negative pulse glial chain, the glia generates the pulse when the glia is excited by the connected neuron. The excitation glia generates the positive pulse when the connected neuron has the large output. The excitation glia generates the negative pulse when the connected neuron has the small output. We consider that the pulse glial chain gives the relationship of position of the connected neurons and that this relationship improves the MLP learning performance. By solving the TSP, we confirm that the MLP with positive and negative pulse glial chain has the high learning performance and the generalization capability. Moreover, it can clearly represent the two spirals.

\section{AcknOWLEDGMENT}

This work was partly supported by JSPS Grant-in-Aid for Scientific Research 22500203.

\section{REFERENCES}

[1] P.G. Haydon, "Glia: Listening and Talking to the Synapse," Nature Reviews Neuroscience, vol. 2, pp. 844-847, 2001.

[2] S. Koizumi, M. Tsuda, Y. Shigemoto-Nogami and K. Inoue, "Dynamic Inhibition of Excitatory Synaptic Transmission by Astrocyte-Derived ATP in Hippocampal Cultures," Proc. National Academy of Science of U.S.A, vol. 100, pp. 11023-11028, 2003.

[3] S. Ozawa, "Role of Glutamate Transporters in Excitatory Synapses in Cerebellar Purkinje Cells," Brain and Nerve, vol. 59, pp. 669-676, 2007.

[4] G. Perea and A. Araque, "Glial Calcium Signaling and Neuro-Glia communication," Cell Calcium, vol. 38, 375-382, 2005.

[5] S. Kriegler and S.Y. Chiu, "Calcium Signaling of Glial Cells along Mammalian Axons," The Journal of Neuroscience, vol. 13, 4229-4245, 1993.

[6] M.P. Mattoson and S.L. Chan, "Neuronal and Glial Calcium Signaling in Alzheimer's Disease," Cell Calcium, vol. 34, 385-397, 2003.

[7] C. Ikuta, Y. Uwate and Y. Nishio, "Chaos Glial Network Connected to Multi-Layer Perceptron for Solving Two-Spiral Problem," Proc. ISCAS'10, pp. 1360-1363, May 2010.

[8] C. Ikuta, Y. Uwate, and Y. Nishio, "Performance and Features of Multi-Layer Perceptron with Impulse Glial Network," Proc. IJCNN'11, pp.2536-2541, Jun. 2011.

[9] D.E. Rumelhart, G.E. Hinton and R.J. Williams, "Learning Representations by Back-Propagating Errors," Nature, vol. 323-9, pp. 533-536, 1986.

[10] J.R. Alvarez-Sanchez, "Injecting knowledge into the Solution of the Two-Spiral Problem," Neural Computing E Applications, vol. 8, pp. 265272, 1999.

[11] H. Sasaki, T. Shiraishi and S. Morishita, "High precision learning for neural networks by dynamic modification of their network structure," Dynamics $\mathcal{E}$ Design Conference, pp. 411-1-411-6, 2004. 